\title{
« Les Grands BufFets », UNE ÉDUCATION COMMENSALE
}

\section{Parcours gastronomique, narrations GOURMANDES ET IMAGINAIRE RABELAISIEN DANS UN RESTAURANT « D'EXCEPTION »}

\author{
Pascal LARDELLIER ${ }^{1}$
}

\begin{abstract}
« Les Grands Buffets » de Narbonne, dont il va être question dans ces pages, constituent un cas unique en France dans l'univers de la restauration. Il s'agit en effet d'un restaurant d'exception qui, entre narrations et discours, concilie les codes de la « cuisine traditionnelle française » et la formule « buffet à volonté ». Étonnant appariement dont cet article se fera l'écho.
\end{abstract}

« La certitude de vivre une expérience unique dans un restaurant qui ne ressemble à aucun autre... »

Pierre Privat, fondateur des « Grands Buffets $»^{2}$.

1 Pascal Lardellier est professeur en Sciences de l'Information et de la Communication à l'Université de Bourgogne Franche-Comté.

2 Citation extraite du porte-folio de prestige (papier glacé, photos en quadrichromie...) présentant « Les Grands Buffets », et mis à la disposition des médias et des partenaires.

Recherches en communication, $n^{\circ} 48$ - Article publié le 14/07/2020 


\section{« Les Grands Buffets » ${ }^{3}$, terrain et discours...}

« Les Grands Buffets » de Narbonne (Aude) constitue un cas unique $^{4}$ en France dans l'univers de la restauration. Il s'agit en effet d'un restaurant conciliant les codes de la cuisine française de haute tradition quant au cadre, au service, aux plats proposés ; mais pour autant, il est organisé sur le principe de la formule « buffet à volonté ». C'est de cet alliage paradoxal dont nous allons prendre la mesure dans ces pages. Car ordinairement, le (très) bon restaurant ne se donne pas "à volonté ", mais de manière parcimonieuse. Quant aux buffets du même nom, qui prolifèrent en zones péri-urbaines, ils ne proposent jamais de la cuisine " de tradition » qui mettrait à l'honneur des recettes appartenant au patrimoine culinaire (français). «Les Grands Buffets » a choisi cette option unique en son genre, d'une cuisine qualitative à volonté. Et les chiffres tendent à prouver le succès de ce restaurant ${ }^{5}$.

C'est dans cet établissement « de tous les superlatifs » que nous avons mené une enquête de terrain, en l'investissant avec les méthodes de l'anthropologue. Si les items contextuels inhérents à l'analyse anthropologique seront mobilisés, il va convenir de donner quelques clés permettant de comprendre comment sa réussite est produite. Elle l'est par des stratégies discursives, déjà : en effet, «Les Grands Buffets » doit

3 Dans ces pages, «Les Grands Buffets », nom commercial, sera mis entre guillemets et conjugué au singulier, en tant que substantif générique et non entité plurielle.

4 « Restaurant d'exception », « unique », « de tous les superlatifs »..., les locutions présentes dans ce texte qui renvoient à ce caractère exceptionnel ne sont pas l'effet du charme ou de la sidération qu'aurait exercé l'établissement étudié sur l'auteur de ces lignes. Ce restaurant est bien « unique » en son genre, au regard de sa taille, de son volume, de son business model, de son positionnement, de son offre, de son chiffre d'affaires et de sa fréquentation. Et ce caractère exceptionnel revient spontanément dans le discours de la plupart des partenaires, des clients et des observateurs des « Grands Buffets ».

5 Des données quantitatives sur « Les Grands Buffets » tendent en effet à justifier cette posture : le restaurant, qui a « le plus gros chiffre d'affaires de France » (14,5 millions d'euros), compte 190 employés, dont 40 cuisiniers, beaucoup de plats étant réalisés « maison et à la minute ». Le site Internet de l'établissement compte un million de visiteurs annuels. Plus de 300 plats sont proposés, et « Les Grands Buffets » comptent par exemple un atelier de flambage (crêpes, tartes...) comptant trois employés à plein temps. Autre chiffre-clé : le restaurant accueille 350000 clients annuels, dont $90 \%$ viennent d'hors de la région Occitanie, où se situe Narbonne. Et on note $10 \%$ de clientèle étrangère. Enfin, il écoule 87000 bouteilles de vin par an. 
son succès à un habile entrelacs de narrations. Celles-ci se complètent, se superposent, pour produire un récit global cohérent et valorisant.

Mais la forte attractivité de l'établissement vient surtout des conditions rituelles et expérientielles offertes lors de la visite en ses murs. L'expérience donnée à vivre là, gastronomique et symbolique, constitue même le « dieu caché » du système. Symbolique disais-je ? Oui, car lors de la visite aux « Grands Buffets », il est question d'identité, de communauté, de tradition et de la célébration de celles-ci, et pas seulement de se restaurer.

Il ne s'agit pas de faire ici la promotion d'un établissement unique en son genre (déjà eu égard au paradoxe mentionné plus haut), mais de comprendre en quoi cette réussite ${ }^{6}$ connaît des ressorts sous-jacents profonds, précisément anthropologiques. Et c'est là que nous souhaitons aller : ne pas cantonner l'analyse à une approche qui s'apparenterait au marketing ou aux sciences de gestion (nous n'en avons pas les compétences), mais « monter» vers les structures symboliques agissant au corps défendant des acteurs. Car « Les Grands Buffets » propose à ses clients d'y vivre une séquence enchantée, ouvrant pour eux une parenthèse spatio-temporelle qui mobilise toutes les dimensions du rite. Et c'est à ce titre que « Les Grands Buffets » n'est pas un restaurant ordinaire : y venir se prépare (réservation obligatoire en ligne longtemps avant), y passer un (long) moment se savoure (et déjà comme expérience), " revivre sa visite » (photos et films à la clef) s'inscrit dans la mémoire personnelle, familiale et sociale... Tout est fait pour que l'expérience soit « hypermnésique », comme savent l'être les rites. Et « hypermnésique » tant du point de vue physique, émotionnel que cognitif, le rite sollicitant toujours autant l'esprit, le corps que les affects. Et on y vit une séquence « liminale » (Turner, 1983), s'inscrivant hors de l'espace et du temps ordinaires, séquence enchantée puisque la paisible opulence et le luxe ambiant confèrent à l'expérience un caractère singulier, rituel mais aussi mythique (célébration de valeurs rabelaisiennes, se matérialisant dans les discours, l'abondance mise en scène, les devises..., nous y reviendrons en conclusion).

6 Cette affirmation n'est pas un jugement de valeur subjectif, mais un état de fait, fondé sur le chiffre d'affaires du restaurant (en millions d'euros), sur les centaines de milliers de clients ayant fréquenté l'établissement, avec un taux de satisfaction record (commentaires élogieux dans le "Livre d'or » ou en ligne, visites régulières...), sur la longueur de la liste d'attente, et la durée en semaines voire en mois, du délai de réservation moyen... Cf. la note précédente sur ces aspects des choses. 
On trouvera donc ici une analyse du dispositif général, dans ce qu'il mobilise comme mythes et imaginaires, mais aussi dans ce qu'il induit comme perception du moment vécu et partagé là. Et nous convoquerons les ressources de l'anthropologie de l'enchantement (cf. bibliographie, Winkin), pertinente pour « lire » « Les Grands Buffets ».

\section{Les nouveaux terrains de l'anthropologie}

Ouvrons la focale : plus largement, ce texte est à placer dans la lignée d'un projet théorique plus large, faisant suite au programme énoncé par Marc Augé dans son ouvrage Pour une anthropologie des mondes contemporains (1990).

Il y expliquait en substance que les « isolats anthropologiques » s'étant raréfiés, l'anthropologue devait se convertir à des objets contemporains. Par nécessité, poursuivait-il, il convient de considérer qu'on ne peut plus astreindre l'anthropologie « aux mirages de la fuite, de l'exil ou de l'exotisme ». Alors, il faut réaffirmer la nécessité épistémologique d'une « anthropologie des mondes contemporains », certes, mais aussi des modes contemporains de représentations sociales, et des formes nouvelles de faire lien.

Au-delà des chantiers ruralistes, conjointement à la conversion récente de ses terrains et de ses objets, et par-delà les analyses précieuses d'un monde exotique désormais situé à nos portes, c'est aussi l'étude d'une nouvelle économie symbolique et relationnelle qui est proposée à l'anthropologue ; et ce afin d'aider à penser, grâce au recul et à la profondeur de cette discipline, des organisations sociales a priori récentes, mais qui reproduisent des modèles anciens, embusqués dans des structures pérennes, derrière leur apparente modernité.

Suite à la parution de son ouvrage-manifeste, Marc Augé a livré une série d'études célèbres pour certaines, qui illustraient son projet : Un ethnologue dans le métro, Un ethnologue à la Coupe du Monde, Un ethnologue à Center Parcs, Un ethnologue à Disneyland...

Eh bien ce travail sur « Les Grands Buffets », l'observation participante menée sur ce terrain, s'inscrit dans la lignée de ce renouvellement auquel invite Augé ; et il se situe, nous concernant, à la suite d'un ensemble d'enquêtes menées, depuis une vingtaine d'années, sur des terrains tels que les Apple stores, le GIGN, les « confréries vineuses » de Bourgogne ou le Festival de Cannes.

La démarche est donc celle d'une recherche engagée, " incarnée » et non de seconde source. Pour cela, prendre le parti d'investir et d'in- 
vestiguer les terrains de l'étude, pour aller au plus près des phénomènes à étudier et des acteurs, et pour voir concrètement « comment ça se passe ». Gageons que nous serons parvenu à « objectiver notre subjectivité "), en remettant les faits et les effets de perception à bonne distance analytique, et en les passant au tamis de la théorisation sémio-anthropologique.

Si le projet général de cet article est clairement anthropologique, la problématisation le rattache aux sciences de l'information-communication; en effet, tout est affaire de médiations aux « Grands Buffets » : médiations sensibles, médiations discursives, médiations symboliques. Et l'on sait l'importance de ce concept pour l'interdiscipline que constituent les sciences de l'information et de la communication, véritable « colonne porteuse » théorique de la discipline.

\section{De la méthode}

Notre enquête s'est appuyée sur une lecture exploratoire de documents de différentes natures sur « Les Grands Buffets », puis d'une demi-journée passée dans le restaurant. Celle-ci a commencé par une rencontre avec Emma Fabbro, attachée de presse de l'établissement, avec qui j'avais préalablement pris contact, en lui expliquant ma démarche ${ }^{7}$. Elle m'a accordé un long entretien, me présentant « Les Grands Buffets » (visite guidée, rencontres avec des collaborateurs, explicitation de la philosophie de l'établissement, avant de répondre à des questions que j'avais préparées en amont. J'ai ensuite pu librement déambuler en observateur participant, puis déjeuner (dans la « Tente d'apparat »), afin de tester la carte et le service, et d'observer l'ensemble du dispositif. J'ai pu ensuite refaire un tour général avant de prendre congé dans l'après-midi. Durant ce temps passé aux « Grands Buffets », j'ai pris des notes et des photos, j'ai discuté avec des clients, et j'ai observé avec attention l'agencement général, l'organisation, les déambulations, les interactions. Après ma visite, j'ai complété ce recueil d'informations par la lecture et l'analyse, de nouveau, des nombreuses sources sur « Les Grands Buffets » disponibles en ligne.

7 Dans le corps du texte, les verbatim tirés de notre entretien sont entre guillemets. Cette visite a eu lieu le 5 août 2019. Toutes les photos illustrant cet article ont été réalisées ce jour-là. 


\section{Un restaurant « superlatif»}

«Les Grands Buffets » est un établissement unique, appuyant donc d'abord son succès sur un entrelacs de discours. Expliquons-nous : J.-M. Adam définit le discours comme « un énoncé caractérisable certes par des propriétés textuelles, mais surtout comme un acte de discours accompli dans une situation (participants, institution, lieu, temps) ${ }^{8} »$. Or, à « lire » « Les Grands Buffets », on perçoit ces discours spécifiques, que nous allons nous attacher à identifier.

Ce restaurant cultive en premier lieu à son propre égard un discours assumé du gigantisme et de l'exceptionnel, puisqu'il s'agit « du restaurant de tous les records ». La chose est dite, écrite (via le site, les dossiers de presse, les articles...) et revendiquée ; et tant les chiffres caractérisant le restaurant que le " Guinness livre des records » attestent de ce « gigantisme »... rabelaisien.

Créé en 1989 par Pierre Privat (« sur le modèle qualitatif des buffets du Club Méditerranée »), le restaurant, eu égard à son standing et à sa notoriété, est implanté dans un emplacement incongru : dans une zone commerciale, juste à côté d'un parc aquatique. C'est par « effet d'aubaine » que le fondateur a pris la décision de s'implanter là, lors d'un appel d'offres précédant la création de cette zone.

Le site Internet des Grands Buffets (https://www.lesgrandsbuffets. $\mathrm{com} / \mathrm{fr}$ ) propose une plongée immersive dans l'univers du restaurant, photos, vidéos et textes animés à l'appui'. Ce site compte plus d'un million de visiteurs par an, et propose un système de réservation (obligatoire) très efficace. Le délai d'attente pour avoir une table varie de quelques jours en basse saison à quelques semaines (voire mois) en haute saison. Il est à noter que "Les Grands Buffets» ne permettent pas de « privatiser » des salles ou de réserver de grandes tables pour des événements tels que mariages et autres fêtes familiales ou professionnelles. Mais beaucoup de repas de famille y sont organisés, et un certain nombre de clients reviennent rituellement une ou plusieurs fois par an, soit parce qu'ils sont de passage dans la région (c'était le cas de mes voisins de table avec qui j'ai pu longuement échanger), ou parce qu'ils « souhaitent honorer cette adresse unique, pour la qualité de sa carte,

8 J.-M. Adam, Éléments de linguistique textuelle, Bruxelles, Mardaga. 1990, p. 23.

9 Il ne nous semble pas pertinent ici de paraphraser ce site ou d'en copier-coller des passages, et nous préférons y renvoyer les personnes intéressées, cette visite complétant à notre sens utilement la lecture de cet article. 
par fidélité envers la maison, son fondateur, ses valeurs » (témoignage d'un client).

Le dispositif général comporte plusieurs « espaces » de restauration, chacun ayant « leur univers »: une brasserie " à la parisienne » cossue, en boiseries et cuivre (photo de gauche); une terrasse extérieure arborée, et une « Tente d'apparat » (photo de droite), qui a ouvert en juin 2019.

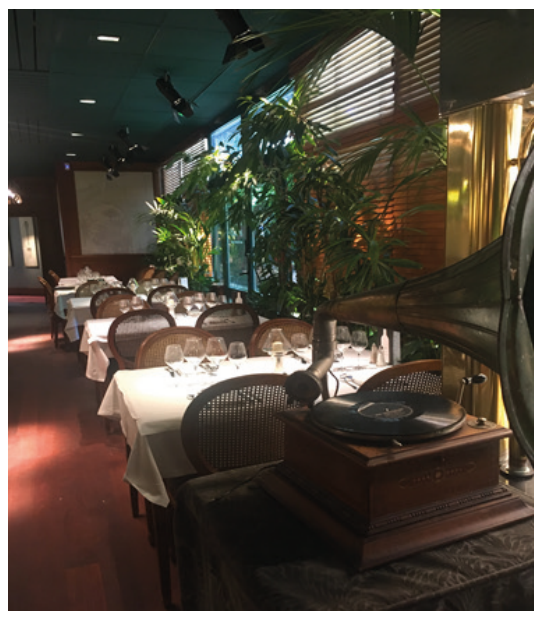

Vue de la partie intérieure du restaurant

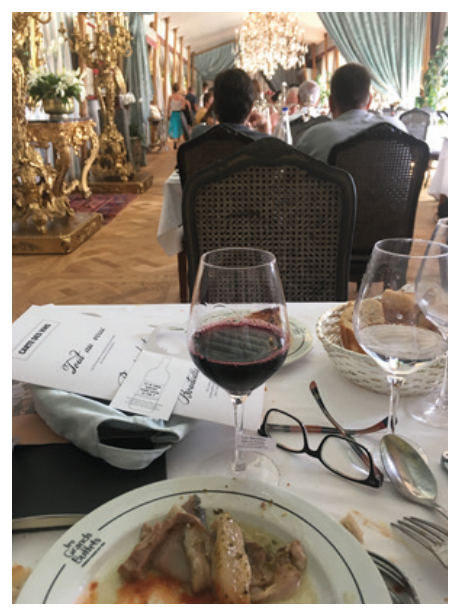

Perspective sur la Tente d'apparat

Une grande cuisine (rôtisserie) panoramique (photo) est sise en face de l'entrée, au milieu du restaurant. Une brigade vêtue de grands tabliers blanc et coiffée de toques s'y active, pour confectionner à la minute les commandes passées par les clients qui attendent en assistant au spectacle. Selon un rituel parfaitement rodé, le chef annonce à haute voix le plat qui vient d'être commandé, et une personne de son équipe s'y affaire aussitôt, après quoi il ou elle se lance dans sa réalisation avec une maestria théâtralisée. Une cloche sonne solennellement quand le plat est prêt, alors que celui-ci est annoncé au micro. Belle trouvaille rituelle que cette cloche, qui dramatise de manière heureuse la remise d'un plat. Le symbolique, la « distinction » (« c'est à mon tour, c'est le mien ») outrepassent la simple fonctionnalité de la séquence.

Parenthèse scénographique : la dichotomie théâtrale canonique entre espace de production (coulisse) et espace de représentation (scène) proposée par E. Goffman (bibliographie) est ici rompue et même inversée, puisque le spectacle n'est pas dans la salle, mais dans la mise en scène des cuisiniers à l'œuvre, et des buffets proposant une profusion 
savamment agencée de plats ordinairement servis à l'assiette en quantité bien moindre et pour cause.

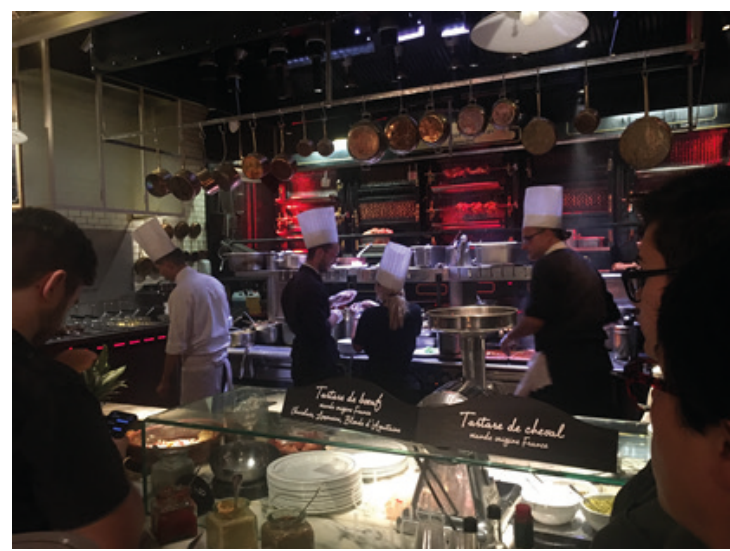

La Rôtisserie, les cuisiniers « en action »

Le projet du restaurant, parfaitement théorisé et explicité, est de proposer un « parcours », parcours tout à la fois gastronomique, expérientiel, et en quelque sorte initiatique dans « les trésors de la "cuisine traditionnelle française", dont "Les Grands Buffets" entendent être un conservatoire » (site Internet de l'établissement). Il est théorisé et explicité, disais-je, car le projet général a été conçu dans ses moindres détails par Pierre Privat, homme d'affaires mais aussi intellectuel et amateur/ collectionneur d'art. De l'inspiration « buffets du Club Med» à la carte comme « conservatoire de la cuisine française » du dispositif général au tarif unique et à la « politique prix » du vin en passant par le choix de l'artisanat et du « local» dans les partenariats (fournisseurs...) et jusqu'aux maximes rabelaisiennes parsemant le restaurant, rien n'a été laissé au hasard. Et l'ensemble compose une mosaïque très cohérente.

Et ce projet est explicité, car les dossiers de presse, le site Internet mais aussi les discours tenus par les employé-es vont dans le même sens, celui de cette tradition culinaire et gastronomique française à faire vivre et honorer en ce lieu singulier.

D'ailleurs, que peut-on savourer dans cet établissement, alors que la tendance actuelle est à une gastronomie métissée, et travaillée par les « interdits » (diététiques, moraux, religieux... $)^{10}$ ? C'est le patrimoine

10 Se reporter à des synthèses telles que celles proposées par François Ascher, Le Mangeur hypermoderne (Paris, Odile Jacob, 2005), Claude Fischler et Estelle Mas- 
culinaire français qui est là réhabilité et mis à l'honneur : tournedos au foie gras, bouchées à la Reine aux ris de veau et morilles, daubes, tripes, blanquettes, civets, cuisses de grenouilles, turbot entier cuit au four, cochon de lait et agneau de lait des Pyrénées, gratins, coquillages, pâtés, homards, et farandoles de desserts (une cinquantaine à la carte)...

Fait remarquable, qui a aussi contribué à la notoriété du restaurant : le tarif (unique) est de 37,90 euros, ce qui donne « accès en illimité » à tous les plats. Le repas est gratuit pour les enfants de moins de 10 ans, et il en coûte 18,90 euros pour ceux de 6 ans à 10 ans.

Tous les fruits et légumes servis sont « bio », la maison privilégie les filières courtes, et sans cesse, des nouveautés en termes de produits, d'offres et de services sont proposées. La philosophie est de proposer « des repas de fêtes, mais tous les jours ".

Autre exception tarifaire : les vins sont vendus à prix coûtant, et ceci fait des «Grands Buffets » le premier prescripteur de vins de France. Le restaurant vend 87000 bouteilles à l'année, mettant particulièrement à l'honneur les vins du Languedoc-Roussillon ${ }^{11}$. L'établissement peut se prévaloir d'offrir « le plus large choix de vins au verre de France ». D'ailleurs, si l'on achète une caisse de 6 bouteilles du vin servi à table, cette bouteille est offerte aux clients.

Notons aussi que « Les Grands Buffets » s'enorgueillissent de proposer le « plus grand plateau de fromages au monde» (photo), le « livre Guinness des records » ayant validé ceci en 2019.

Le business model est celui-ci : " une marge faible, une rationalisation extrême via un système de réservation en ligne permettant de savoir en temps réel le nombre de couverts et de plats à prévoir. De ce fait, les quantités se trouvent ajustées au plus près ». Car la question du gaspillage est saillante aux "Grands Buffets », tout est ajusté par réservation et de savants calculs (ainsi, « 1,8 huître par personne »), et tout est produit sur place. Quant aux collaborateurs, ils sont nourris au restaurant, mais avec les plats qui ne sont plus présentables (« quand il ne reste plus que deux parts de tarte, on ne peut pas laisser cela pour la clientèle ; alors c'est pour le personnel »).

son, Manger. Français, Européens et Américains face à l'alimentation (Paris, Odile Jacob, 2008) ; Claude Fischler (dir.), Les Alimentations particulières. Mangerons-nous encore ensemble demain ?, (Paris, Odile Jacob, 2013), Michel Wieviorka (dir.), Se nourrir. L'alimentation en question (Auxerre, Éditions Sciences Humaines, 2009).

11 « La bouteille de Cigalus de chez Gérard Bertrand est proposée à 28 euros, la même valant 200 euros au Jules Verne à Paris ». 


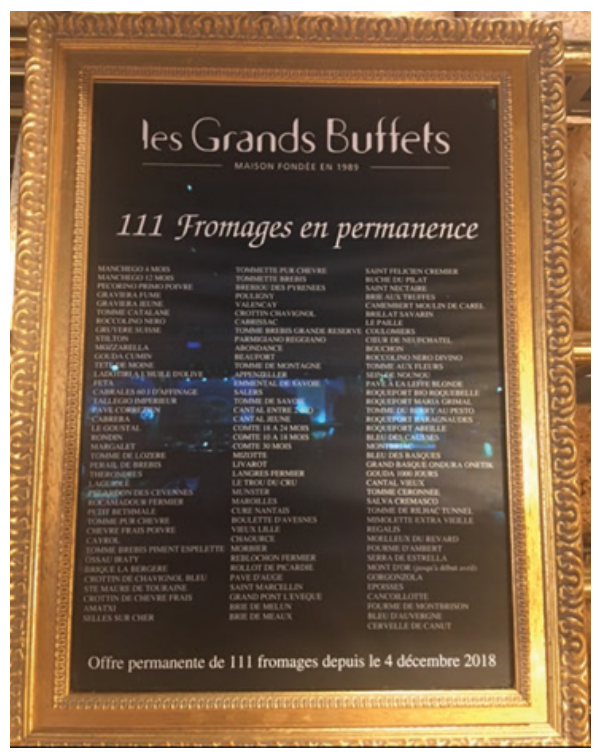

La carte des fromages inscrite au Livre « Guiness des records »

En clair, le positionnement général des " Grands Buffets » prend le contrepied de tous les buffets à volonté (souvent asiatiques) ayant fleuri depuis une vingtaine d'années en périphéries des villes, et moins préoccupés (euphémisme) par le raffinement des mets et la célébration des « arts de la table » que « Les Grands Buffets ».

S'il n'y a « pas d'étude officielle sur la sociologie de la fréquentation des « Grands Buffets », notre clientèle est diverse avec une nette progression depuis plusieurs années sur un public « CSP+ » et un rajeunissement de la cible (moyenne 40 ans)... Par ailleurs, nous progressons avec $50 \%$ actuellement de nouveaux clients par mois. Non pas que la fidélisation baisse, mais le pourcentage de nouveaux clients augmente car nous sommes en capacité d'accueil maximale » (entretien avec Emma Fabbro).

En tout cas, il ne s'agit pas là de « manger jusqu'à plus soif, ni de faire de l'ingurgitation une performance ${ }^{12} »$, comme dans les autres buffets à volonté. Aux « Grands Buffets » (où on parle de « manger à discrétion »), on perçoit une ambiance générale assez feutrée, des déambulations des clients plutôt lente, peu voire aucune queue devant certains buffets (sauf une petite file d'attente devant la rôtisserie pano-

12 Pascal Lardellier, Manger jusqu'à plus soif... Les buffets à volonté, résurgences postmodernes du pantagruélisme, Opéra bouffe. Une anthropologie gourmande de nos modes alimentaires, Caen, EMS, 2013, pp. 121-139. 
ramique). Et surtout, on est servi à table en vin et en eau, les assiettes sont desservies, une relation personnalisée peut se nouer avec l'employé assigné à sa table. En clair, la logique du « glouton », qui peut être de mise ailleurs, est ici ravalée au rang de pratique dégradante ; aux « Grands Buffets », on souhaite flatter le gourmand, et même avoir des gourmets comme convives.

Revenons à ces discours qui se complètent pour finalement produire « Les Grands Buffets » comme un « lieu d'exception ».

\section{Un discours corporate, institutionnel et médiatique}

Pleinement conscients de leurs particularités, « Les Grands Buffets » appuie son succès et sa notoriété sur une stratégie discursive raisonnée, se déployant dans plusieurs directions, et selon plusieurs canaux. Plusieurs directions, c'est-à-dire vers les clients, vers les prescripteurs (médias) et vers les institutions (Région Occitanie, syndicats professionnels...) ; et plusieurs canaux, via la communication dans l'établissement, mais aussi par les plaquettes, dossiers de presse, articles et reportages, prolongements numériques (site...).

Dès l'entrée du restaurant, le visiteur découvre un mur où sont apposés (photo) de petits panneaux de légitimation : Petit Futé, Gault et Millau...

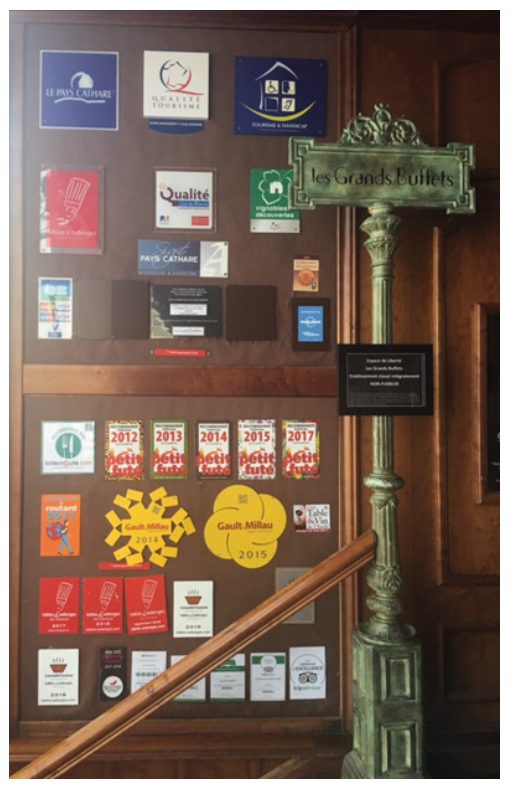

Les multiples plaques de

recommandation à l'entrée du restaurant 
Il est de bon ton, pour un établissement, d'asseoir sa réputation sur des discours de légitimation, tenus en direction de ses clients et partenaires ; mais aussi de diffuser ces messages via les relais médiatiques et numériques. Ainsi en va-t-il d'abord du site Internet des " Grands Buffets », extrêmement bien conçu, avec galerie de photos, vidéos... Dans le Livre d'or, les témoignages d'inconnus côtoient ceux de « stars » (Christian Clavier, Pierre Arditi...). Et tous célèbrent cette adresse unique, pour laquelle on est prêt à attendre des semaines voire des mois, et à faire des centaines de kilomètres.

Et il est clair que « Les Grands Buffets », au regard de son caractère unique et de son paradoxe fondateur (une cuisine très qualitative, « de tradition », à volonté), constitue un sujet de choix pour les médias. Et le book de l'établissement comprend des dizaines d'articles, journaux, magazines et télévisions lui ayant consacré des reportages souvent élogieux (la plupart ont été mis en ligne sur le site du restaurant). Les « grandes plumes » de la critique gastronomique autant que les « défenseurs du bien manger » ont écrit sur l'établissement, de Périco Légasse au magazine Thuriès Gastronomie.

\section{Un souci pédagogique constant}

Posture assez singulière, "Les Grands Buffets » adopte un discours étonnamment didactique en direction de ses clients, via un éventail d'explications disséminées sur maints supports quant au fonctionnement du restaurant proprement dit, à ce qui est proposé, et plus largement quant à sa philosophie.

L'établissement peut se prévaloir de l'édition de règles strictes en amont de la visite, quant à la réservation en ligne, puis in situ par une attente derrière des cordons contribuant à la solennité du lieu. Nul n'entre là sans y avoir été convié, sans y être attendu, sans y être pris en charge ; précisément comme dans un lieu symbolique où l'on procéderait à une initiation... Et dès l'entrée, des panneaux d'avertissement et des règles de bonne conduite sont affichés, dont le visiteur doit prendre connaissance. Encore une fois, on se trouve aux antipodes des buffets à volonté ordinaires, où aucune de ces précautions n'est affichée, et où prévalent une standardisation et une taylorisation seules garantes de la rentabilité du modèle.

Ensuite, fait unique, un parcours guidé de quelques minutes est proposé par une employé·e à tous les nouveaux clients, afin de leur présenter l'établissement et ses différents « univers », mais aussi « afin 
que les convives ne ressentent pas " la crainte de ne plus en avoir » (sic). Bref, il n'y a aucun lieu de se précipiter. La lenteur et la courtoisie devant présider aux déambulations en ce lieu sont induites par cette visite d'initiation.

Ce « parcours du goût » sert à initier, tout en présentant l'éventail de ce qu'il sera possible de déguster. Je l'ai moi-même réalisé avec Emma Fabbro : on déambule dans le restaurant et on découvre les univers proposés, « La Mer », " La Rôtisserie », « les Plats canailles » (photo). L'humour n'est pas persona non grata. Ainsi, voit-on une plaque : « Fromages qui puent »...

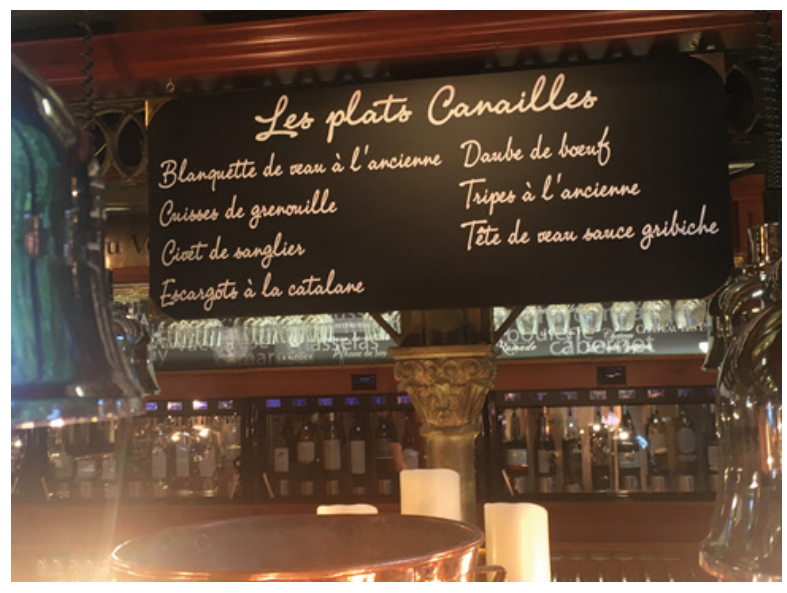

Tableau des «plats canailles »

Ensuite, « Les Grands Buffets » affirme cette posture par une série d'items informatifs : les salles sont parsemées de plaques et de cartels en fer gravé permettant d'identifier les différents espaces (« Recettes canaille », « Recettes de grand-mère »), mais aussi les plats, tous clairement désignés et explicités par de petits « didacticiels ${ }^{13}$ » (fromages, vins..., cf. photos). Au dispositif général du restaurant, se superpose un métadiscours signalétique (panneaux laiton, cartels..) permettant d'identifier clairement les dénominations, les origines, les millésimes, les labels...

13 Ce mot, employé en muséographie et en médiation culturelle (sensiblement synonyme de cartel, avec une visée pédagogique) a été employé par Emma Fabbro à plusieurs reprises lors de notre entretien, pour qualifier les petites plaques descriptives et explicatives « sous-titrant » les plats en présentation. 


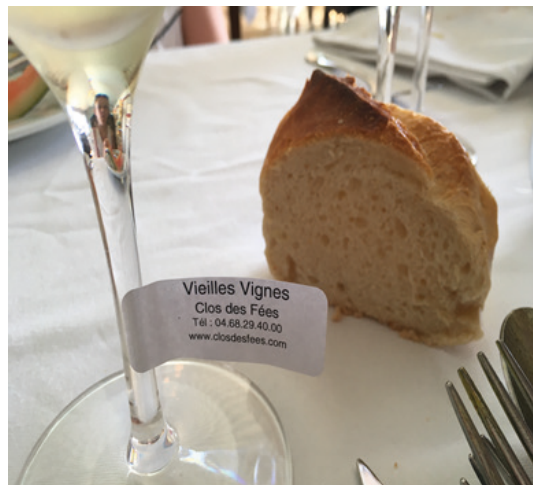

Gros plan sur les étiquettes de dégustation du vin

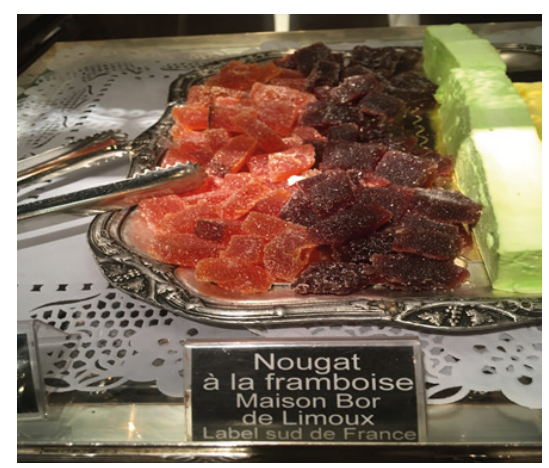

Chaque plat est nommé, identifié, référencé

L'idée est d'œuvrer à une « éducation du goût », via ce " parcours initiatique » dans les traditions gastronomiques régionales, en proposant au grand public des « produits d'exception » issus des régions françaises.

Ainsi, neuf jambons crus sont proposés, avec des affinages différents. De même, un large éventail des huîtres produites en France est représenté, toutes étant dûment étiquetées, classées. Et pas moins de 9 foies gras sont référencés, permettant de tester, de comparer...

Le personnel, très bien formé, prolonge ce dispositif d'explicitation en donnant toutes les indications dont le client aurait besoin (accord mets/vins, origines des produits, mais aussi anecdotes sur les producteurs ...)

\section{Plus rare, un discours sociétal et citoyen}

La philosophie du fondateur des « Grands Buffets » n'est pas faite que de mots, de déclarations d'intention qui ne seraient pas appuyées sur des faits. Ainsi, le restaurant poursuit une politique de valorisation des artisans (locaux), de collaboration avec les producteurs de la région, et de mise à l'honneur des acteurs des filières « bio ». De même, les couverts et tous les accessoires de table relèvent d'un service de standing. Cette veine « éco-responsable », citoyenne, sociétale est un parti pris délibéré. On la retrouve dans les modalités de recrutement du personnel, s'apparentant à une cooptation plus qu'à une sélection, mais aussi dans les horaires de travail privilégiés ( 3 jours de repos par semaine) ou encore un système de prêt d'argent avantageux pour le personnel... 
Pour la réalisation de la « Tente d'apparat » qui a ouvert en juin 2019, il a majoritairement été fait appel à des artisans locaux, ou des spécialistes reconnus : elle a coûté 2,2 millions d'euros de travaux, dont 1,9 dépensé en local. "Il aurait été facile de confier le chantier à Vinci, mais on a tenu à faire travailler les artisans et les petites entreprises et autres PME de la région ». L'entreprise ayant réalisé les lustres est celle qui a ouvragé ceux du château de Chambord. Pour les parquets, c'est l'entreprise ayant réalisé ceux de Versailles. Quant au « mur végétal », il a été laissé aux soins des artisans qui ont arboré celui du Musée du Quai Branly à Paris.

Pierre Privat, féru d'art (et notamment du mouvement de la « figuration libre »), a choisi d'orner son restaurant de nombreuses œuvres d'art. Plus largement, il a choisi de faire de son établissement un « musée des Arts de la Table », ou un « sanctuaire » de pièces françaises rares ou historiques.

Ainsi, a été achetée aux enchères la célèbre " presse à canards » de la Tour d'Argent à Paris. Et cette presse n'est pas décorative, mais elle sert à proposer ce plat (le « canard au sang »), entouré de son cérémonial. Autre acquisition, l'atelier de flambage du Negresco (photo).

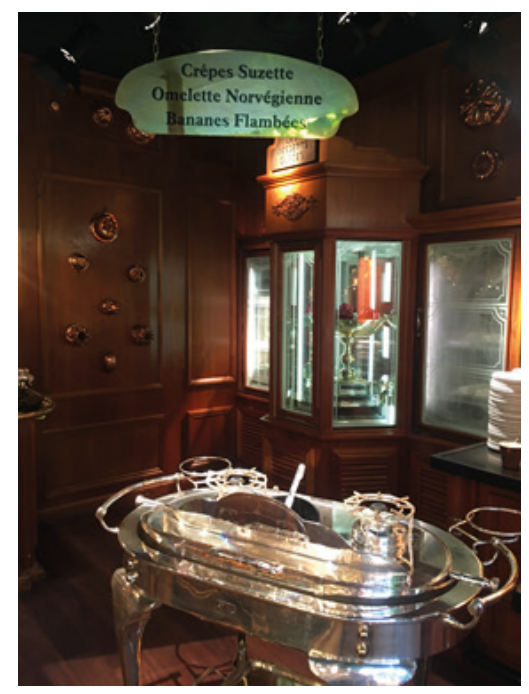

L'atelier de flambage en argent, acheté au Negresco (Nice) 
Ces pièces historiques dépassent la logique d'exposition muséale, puisqu'elles servent au quotidien. Cette volonté de patrimonialisation et de muséification ne se résume pas à une collection d'objets, et beaucoup de « maîtres » (écailler, cuisinier, rôtisseur, saucier, charcutier, crémier affineur, boulanger, pâtissier, chocolatier, confiseur...) officient aux « Grands Buffets », de même que des « MOF » (« meilleurs ouvriers de France ») qui sont représentés parmi les collaborateurs.

\section{La visite aux « Grands Buffets », une séquence rituelle, une parenthèse enchantée}

Proposons une ouverture et une mise en perspective anthropologique.

En fait, la visite aux « Grands Buffets » relève d'une séquence rituelle, en même temps que le moment que l'on s'y donne à vivre (ensemble) projette dans une séquence «d'enchantement». Le rite est une « parenthèse » sociale se caractérisant par sa dimension symbolique et spectaculaire, son caractère réglé, institué, et le fait qu'on (y) célèbre quelque chose ${ }^{14}$. Ou, pour le dire comme Martine Segalen, « le rite est un ensemble d'actes formalisés, expressifs, porteurs d'une dimension symbolique. Il se caractérise par une configuration spatio-temporelle spécifique, par le recours à une série d'objets, par des systèmes de comportements et de langages spécifiques, par des signes emblématiques dont le sens codé est l'un des biens communs d'un groupe ${ }^{15} \gg$. Plus largement, les auteurs mettent en évidence la dimension formelle et symbolique des séquences rituelles, ainsi que leur caractère spectaculaire et narratif. Le rite raconte une histoire. Et « Les Grands Buffets » prennent leur source narrative, scénographique et légendaire dans l'œuvre de François Rabelais, très explicitement (cf. la conclusion à venir).

Solennels, théâtraux, spectaculaires, symboliques, les rites sont aussi des moments de partage et de plaisir, qui font entrer dans une autre dimension, en créant des conditions mentales et sociales particulières. Le rite est souvent articulé à un mythe, récit fondateur enchantant les origines. Or, on retrouve en tous points dans la visite aux « Grands Buffets » les éléments de cette définition. En effet, on doit préparer la visite par une réservation en ligne, une (longue) attente est imposée,

14 Cf. Pascal Lardellier, Sur les traces du rite. L'institution rituelle de la société, ISTE, Londres, 2019, pp. 26-29.

15 Martine Segalen, Rites et rituels contemporains, Paris, Nathan, 1998, p. 20. 
et le déplacement vers le site constitue souvent un trajet que d'aucuns pourraient considérer comme un «pèlerinage ». Une fois arrivé(s) sur place, des règles strictes vont organiser le temps qui y est passé : on attend en file derrière des cordons, les «néophytes » ont droit à une visite guidée qui « initie » à la géographie et à l'agencement général $\mathrm{du}$ lieu. On va y vivre un moment unique (festif, amical...) qui « fera date et souvenir », un moment « hypermnésique », comme savent l'être les rites, séquences situées hors de l'espace et du temps, s'inscrivant dans la mémoire émotionnelle et marquant celles et ceux partageant le moment pour produire de l'appartenance, réaffirmer une identité, refonder des liens communautaire (souvent familiaux là). Nombre de clients réalisent d'ailleurs des films et font des photos ou des selfies, pour garder traces du moment « unique » vécu là.

Le dispositif général induit cela, puisque la mise en valeur générale des plats et des mets, le ballet cérémonieux du personnel, les annonces, la noblesse des matériaux de l'établissement (bois, cuivres, laiton...), tout cela procède d'une scénographie (or, tout rite prend naissance et sens dans un décorum), et même d'une dramaturgie, paisible et opulente. Les documents explicitant la philosophie des «Grands Buffets » (dossiers de presse, site...) en appellent aux « rituels des arts de la table », au « cérémonial du service à la française », et au « repas français inscrit au patrimoine immatériel de l'UNESCO » depuis 2010.

D'ailleurs, l'écrasante majorité des clients viennent en famille ou en groupe amical, et ceci confirme l'idée du repas de fête, du déjeuner ou du dîner rituel. " Les personnes seules sont très marginales, notre clientèle se compose de clients particuliers qui viennent en couple, en famille et entre amis. Nous limitons volontairement l'accueil des groupes au profit des particuliers. Et la durée moyenne est de 2,5 heures passées à table » (Emma Fabbro). Une tablée familiale ou amicale, la durée conséquente passée sur place, ceci confirme le caractère rituel du passage dans cet établissement unique. En effet, le rite rassemble toujours une communauté autour d'un moment qui se célèbre et se recentre à cette occasion, ceci s'inscrivant dans une temporalité particulière, qui ralentit le cours ordinaire des choses. Précisément, on y revient, ce que donne à vivre « Les Grands Buffets ». Et élément important, bien des rites prennent la table, la commensalité, le partage alimentaire comme théâtre symbolique !

Quant à la localisation des "Grands Buffets » autant que sa conception architecturale, elles instaurent l'idée d'une insularité. $\mathrm{Au}$ milieu d'une zone commerciale comme les périphéries en comptent 
toutes, on voit « de loin » les enseignes de magasins de chaînes ou le parc aquatique adjacent, alors qu'on est là à « l'écart du monde », le temps de quelques heures ; on monte quelques marches, une lourde double porte opaque tient lieu d'entrée imposante, on est accueilli par des majordomes, l'établissement est entièrement enclos.... Le lieu « mythique », sursaturé de sens, fermé sur son unicité, est isolé au milieu de «non-lieux » indifférenciés... C'est bien l'idée d'insularité qui s'impose quand on arrive et découvre «Les Grands Buffets », eu égard à ces éléments matériels et spatiaux dûment codés.

La séquence rituelle qu'on vit là est puissamment expérientielle, puisqu'aux « Grands Buffets », tous les sens des convives vont être sollicités, mis en éveil. Le parcours expérientiel (goûter à tout (ou presque) relève d'une déambulation gourmande, d'un cheminement sensoriel, l'indéniable festival des saveurs étant complété par des éléments d'information qui prêtent aux convives une juste intelligence de ce qu'ils savourent, en l'inscrivant dans une narration identitaire plus large (les « bons produits des terroirs de France »).

L'expérience, polysensorielle, se situe dans l'assiette, mais aussi dans le décorum, via la déambulation cérémonieuse des convives « d'univers en univers », de buffet en buffet. Et elle concerne aussi les « arts de la table », et l'intégration ou la réappropriation des codes du « grand restaurant », quittant là leur orbe élitiste pour se trouver démocratisés.

In fine, le rite est une forme symbolique qui offre aux communautés y venant un triple principe, tout à la fois de scénarisation, de dramatisation et d'esthétisation du moment qu'on y vit. Eh bien l'on peut dire que « Les Grands Buffets » permettent aux familles et groupes d'amis y venant de rencontrer ces trois instances : on scénarise, car la visite est préparée puis vécue comme un moment particulier, à raconter ; on dramatise car ce n'est pas un moment ordinaire, mais une séquence exceptionnelle, conviviale mais solennelle, aussi ; le dispositif général offre enfin une esthétisation (beaux matériaux, jardins intérieurs, « Tente d'apparat », nappes blanches et « service à la française »), dont on gardera trace via les photos et les films, montrés, postés, partagés...

Plus largement, la visite aux «Grands Buffets » relève d'une séquence enchantée, cet enchantement étant autant «produit» par la magie du lieu que par les conditions d'accès (attente, voyage...) et le fait qu'on soit dans un lieu, en ayant l'intuition ou la conscience de cela. On vient vivre aux « Grands Buffets » « le souvenir de ses rêves » (expression de Marc Augé). 
Dans un texte fondateur paru en $2002^{16}$, Yves Winkin propose les termes d'une « anthropologie de l'enchantement», terme qu'il exhume des écrits du poète anglais Samuel Coleridge, qui à propos du théâtre, évoquait une "suspension momentanée de l'incrédulité » (willing suspension of disbelief) ; mais cette locution adaptait aussi la « subjonctivité » de Victor Turner, moments sociaux de grâce, qu'offre notamment le rite. Il s'agit, à un moment donné, durant une séquence sociale particulière, d'effectuer un travail de dénégation, pour ne pas voir les termes économiques, logistiques, techniques d'une activité ou d'une expérience « donnés à vivre », et de les « recadrer » en termes « d'enchantement ». « On sait bien mais quand même »... Ainsi en va-t-il de la visite à Disneyland ou à Las Vegas, de nos balades dans des centresvilles piétonnisés et patrimonialisés ou des quartiers muséifiés (cf. les touristes asiatiques à Montmartre sur les traces d'Amélie Poulain), ainsi en va-t-il de ces séquences ostensiblement touristiques dans lesquelles on est pris, mais où l'on « joue le jeu », parce qu'en « garder un bon souvenir » est à ce prix.

«La notion de suspension mérite d'être travaillée, parce qu'elle permet de pointer plusieurs caractéristiques de l'enchantement... Si nous vivons d'ordinaire à l'indicatif (les choses sont ce qu'elles sont), il arrive parfois que nous glissions temporairement dans une vie « liminale », une vie au subjonctif (les choses deviennent ce que nous avions rêvé qu'elles puissent devenir). C'est le temps du Carnaval, bien sûr ${ }^{17}$ »; Y. Winkin propose une liste de contextes et de lieux relevant de cet enchantement : les lieux conçus d'emblée pour enchanter le visiteur (Disneyland...) ; les lieux construits dans un autre but, mais recadrés de manière permanente dans une perspective d'enchantement (centresvilles reliftés); les lieux investis par une opération collective temporaire (festival, Carnaval, spectacle...). Mais il va plus loin : « il s'agit le plus souvent d'espaces-temps circonscrits ; le périmètre à l'intérieur duquel l'opération de suspension prend place est très explicite... Les rapports sociaux reposent sur une relative égalité entre tous et sur un relatif anonymat ${ }^{18} »$. À bien des égards, la visite aux « Grands Buffets » est vécue comme un moment « liminal », un événement personnel, familial et

16 Yves Winkin, Propositions pour une anthropologie de l'enchantement. Dans Paul Rasse, Nancy Midol \& Fathi Triki, Unité-diversité : les identités culturelles dans le jeu de la mondialisation, Paris, L'Harmattan, 2002, p. 172.

17 Ibid., p. 172.

18 Ibid., p. 179. 
social particulier. On fait abstraction de l'aspect économique (dont on connaît la règle, fixée à l'avance : un tarif unique), on déambule lentement d'univers en univers, tout à la célébration de la « cuisine de tradition française ", avec, en filigrane de cette opulence sans limites, des imaginaires renvoyant à Cocagne, ou à ces fêtes antiques ou médiévales (Saturnales, fêtes de fous, Entrées royales...) durant lesquelles la nourriture se donnait à foison, comme pour contrecarrer la dureté voire les disettes du temps ordinaire.

\section{« Fay ce que vouldras », Rabelais...}

Le succès des « Grands Buffets », appuyé sur l'excellence culinaire dont peut se prévaloir cet établissement (et sur un rapport qualité/prix intrinsèquement exceptionnel), se fonde aussi sur cet enchevêtrement de discours de différentes natures, qui se complètent, se relaient, se prolongent pour construire un ensemble cohérent; et, nous venons de le voir, sur cette expérience rituelle, sur cette immersion expérientielle...

Or, la narration qui chapeaute toutes les autres aux «Grands Buffets » est celle qui invoque l'esprit rabelaisien semblant prévaloir à toute célébration commensale, dans la culture française. La clef de voûte narrative réside en la promotion de la table française traditionnelle, table métaphorique et métonymique exaltée par Rabelais et le pantagruélisme. D'ailleurs, l'adage « Fay ce que vouldras » trône en lettres majuscules comme une devise de table (photo), et plus encore, de vie ; et symboliquement, comme la signature et la caution de l'œuvre de Pierre Privat.

Mais on est aux « Grands Buffets » en présence d'un Gargantua civilisé, policé, rompu aux codes des salons, où la qualité des mets rejoint le plaisir des mots. Le mythe sous-jacent au succès de ce restaurant caractérisé par son gigantisme (tel Gargantua ou Pantagruel, les célèbres personnages de Rabelais), réside finalement en l'alliance à laquelle il parvient, l'équilibre, l'harmonie trouvés entre l'esprit du pays de Cocagne, et celui de l'abbaye de Thélème... 


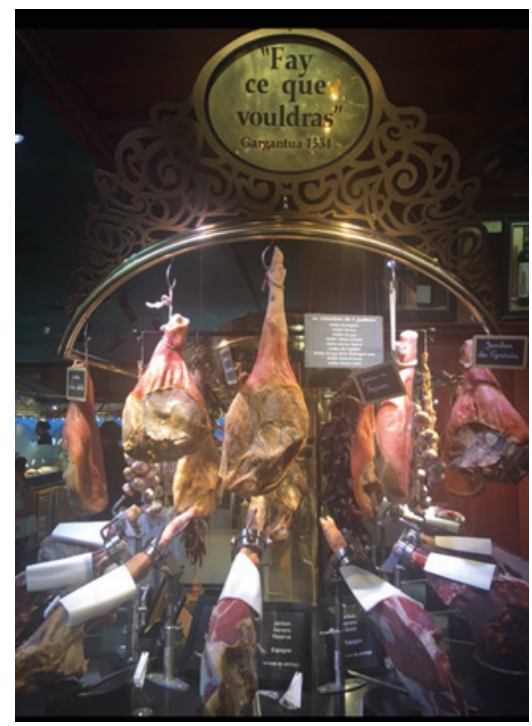

La devise rabelaisienne des « Grands Buffets », face à l'entrée, couronnant un bouquet de jambons

\section{Références}

Ascher, François (2005). Le Mangeur hypermoderne. Paris : Odile Jacob.

Augé, Marc (1994). Pour une anthropologie des mondes contemporains. Paris : Aubier. Bourdieu, Pierre (1979). La Distinction. Critique sociale du jugement. Paris : Les Éditions de Minuit.

Boutaud, J.-J. (dir.) (2004). L'Imaginaire de la table. Paris : L'Harmattan.

Boutaud, J.-J. (2005). Le Sens gourmand, De la commensalité - du goût - des aliments, Paris : Jean-Paul Rocher éditeur.

Boutaud, J.-J. \& Lardellier, Pascal (dir.) (2011). Sémio-anthropologie du sensible, Degrés, Bruxelles, 113.

Fischler, Claude (1990). L'Homnivore. Paris : Odile Jacob.

Goffman, Erving (1973). La Mise en scène de la vie quotidienne. 1. Les rites d'interaction. Paris : Les Éditions de Minuit.

Goffman, Erving (1974). La Mise en scène de la vie quotidienne. Les relations en public, Paris : Les Éditions de Minuit.

Kaufmann, Jean-Claude (2005). Casseroles, amour et crises. Ce que cuisiner veut dire, Paris : Armand Colin.

Kaufmann, Jean-Claude (2007). Famille à table sous le regard de Jean-Claude Kaufmann. Paris : Armand Colin.

Lardellier, Pascal (2011). Opéra bouffe. Une anthropologie gourmande de nos modes alimentaires. Cormelles le Royal, EMS.

Lardellier, Pascal (2019). Sur les traces du rite. L'institution rituelle de la société, Londres, ISTE. 
Onfray, Michel (1955). La Raison gourmande. Philosophie du goût. Paris : Grasset.

Onfray, Michel (1989). Le Ventre des philosophes. Critique de la raison diététique, Paris : Grasset.

Segalen, Martine (1998). Rites et rituels contemporains. Paris : Nathan.

Turner, Victor (1983). The Anthropology of Performance. New York : P.A.J. Publications.

Winkin, Yves (2002). Propositions pour une anthropologie de l'enchantement. Dans Paul Rasse, Nancy Midol \& Fathi Triki, Unité-diversité : les identités culturelles dans le jeu de la mondialisation (pp. 169-179). Paris : L'Harmattan. 